\title{
Formación y desarrollo de la competencia orientación educativa familiar en los docentes
} de educación básica superior

\section{Competencia educativa familiar en la básica superior}

Leonor Alexandra Rodríguez Álava Mg Sc.

Universidad Técnica de Manabí, Ecuador

\section{Contacto: alexanroa32@hotmail.com}

Receptado: 03/03/2015 Aceptado: 10/05/2015

\section{Resumen}

El objetivo de esta investigación fue analizar la necesidad y fundamentos epistemológicos de la formación y desarrollo de la competencia orientación educativa familiar en los docentes de educación básica superior en el proceso de enseñanza-aprendizaje en la unidad Educativa ITSUP, Manabí, Ecuador. En el se destaca la importancia de la relación de la escuela con familia en la formación integral de los adolescentes de este nivel. Para la puesta en marcha de esta experiencia se encuestaron 65 padres y 16 profesores. Los resultados del cotejo probaron que existe un bajo interés por la colaboración de los padres con la escuela, de la misma forma se pudo constatar que los profesores poseen una baja preparación para enfrentar esta relación con los representantes de los estudiantes. Se concluyó que para que exista un fortalecimiento entre los componentes de la escuela y la familia, debe trabajarse en el proceso de formación y práctica de esta competencia.

Palabras clave: Orientación educativa familiar, competencias, relación, desarrollo, familia, integral.

\section{Training and development of educational guidance familiar competition in basic education teachers top}

\begin{abstract}
The objective of this research was to analyze the need and the epistemological foundations of the formation and development of family educational-orientation competence in the teachers of the higher basic education during the academic process in the educational unit ITSUP, Manabi, Ecuador. It is stated in the work, the outstanding importance of the relationship between the school and the family in the formation of adolescents at this level. For the implementation of this experiment 65 parents and 16 teachers were interviewed. The results
\end{abstract}


of the inquiry proved that there is low interest of parents involved with the school affairs. In the same way; it was found that teachers are underprepared to face the relationship with students' representatives. It was concluded that there is a need of the strengthening of both, the school and the family and that there should be a work towards the formation and practice of this competence.

Keywords: Educational-orientation competence, competence, relationship, development, family, integral.

\section{Introducción}

La formación docente ha sido preocupación desde los orígenes de la educación, la que se ha potencializado en la actualidad ante los profundos cambios que se han operado a partir del desarrollo científico-tecnológico, la globalización del conocimiento y las aceleradas variaciones del contexto donde se desarrolla la vida humana. Esta es necesaria para lograr la formación integral de los estudiantes y que estos no solo posean la preparación para vivir en su época, sino también que sean capaces de transformar el entorno que los rodea. Todo esto ha traído modificaciones en las concepciones de la educación y de sus parámetros de calidad.

Como se puede apreciar, la formación de los docentes está en el centro de las políticas y debates educativos contemporáneos en función de contar con maestros competentes en la enseñanza básica superior para lograr una educación que responda a los estándares de eficacia establecidos en el presente siglo. Según Vaillant (2008) en la mayoría de los países del contexto latinoamericano, la formación de profesores del nivel secundario se caracteriza por conferirle mayor importancia a lo disciplinar, siendo la preparación pedagógica tardía y secundaria, lo que precisa la necesidad de la formación de competencias docentes, de manera específica, aquellas competencias que le permitan fortalecer la relación escuela - familia.

A través de la historia, se reconoce a la familia y a la escuela como los dos sistemas más influyentes en el desarrollo personal del individuo; ambas son sistemas insertos en un contexto social (Ríos, 2009), y deben dar respuesta a las necesidades que produce una sociedad en cambio; entonces, es lógico pensar que desde las instituciones se promueva un acercamiento entre ambas para incidir sobre los jóvenes de forma coordinada y complementaria; sin embargo, tanto en la educación primaria como secundaria se considera la orientación educativa relacionada con las dificultades de aprendizaje y en menor medida, pero con un grado de necesidad alto, la orientación del desarrollo personal y familiar. En ese orden, es necesario partir de la perspectiva holística de la orientación e insertar al profesor en 
ese proceso. Según Jaik (2011) es necesario que un docente esté en condiciones de actuar para resolver problemas con idoneidad, mejoramiento continuo y ética.

En relación con lo que se mencionó anteriormente se planteó como objetivo: analizar la necesidad y fundamentos epistemológicos de la formación y desarrollo de la competencia orientación educativa familiar en los docentes de educación básica superior. De ahí la importancia de esta investigación para fortalecer la relación de los docentes con la familia.

\section{Principales aspectos teóricos}

Cada vez es menos frecuente que un profesor limite su esfera de actuación simplemente a exponer un tema en clase. El avance del conocimiento y la innovación en los modelos pedagógicos están obligando al ejercicio de más de un papel en la docencia: facilitador del aprendizaje, tutor, orientador educativo, diseñador de materiales didácticos, elaborador de instrumentos de evaluación, asesor para padres, guía de colegas principiantes; adicional a esto, debe cumplir con la tarea de luchar con aspectos que dificultan la labor educativa entre los que se encuentran los problemas sociales de los estudiantes ; abuso de drogas y violencia; el fracaso escolar, la crisis de la educación en la familia y la influencia que ejercen los coeducadores electrónicos, entre otros.

En múltiples ocasiones, los docentes comprueban que por falta de formación adecuada y de tiempo, no pueden ocuparse suficientemente de estos problemas porque difieren por completo de las tareas de enseñanza tradicionalmente asignadas a la profesión. Este profesional requiere de una nueva cultura para lograr excelencia en su desempeño, una cultura que promueva su actualización conforme al contexto social, porque el desempeño del docente debe llevarse a cabo con orden y organización, estar preparado para enfrentar y resolver imprevistos, anteponer el comportamiento amable, reflejar la buena educación en todos sus actos y lograr con el ejemplo cambio de comportamiento (Medina y Barquero, 2012)

Dada la importancia de este aspecto, desde la perspectiva científica, en la última década autores y organismos como: Carmona (2008), Escudero (2009), García, y Maquilòn, (2010), Mc Pherson (2000), Perrenoud (2008), Zabalza (2007), Marchesi, y Díaz, (.2009)entre otros, han abordado la formación de los docentes de todos los niveles de la educación, tanto en su formación inicial, como en aquellos que se encuentran en ejercicio, donde se hace 
especial énfasis en la necesidad del desarrollo de competencias docentes, en función de la calidad educativa.

Tal como se ha mencionado, el docente requiere de un proceso integral de formación en competencias. Según (Medina y Barquero, 2012) el proceso de aprendizaje para la construcción de las competencias es complejo, fundamentado en que los conocimientos se comprenden, las habilidades se desarrollan, las capacidades se construyen, los valores se generan y las competencias se demuestran con el desempeño en la acción y se sujetan a evaluación y certificación con el fin de lograr el resultado esperado.

En correspondencia a la formación de competencias en los docentes, se asume como competencias la definición desde la perspectiva de la socio-formación al considerarlas como actuaciones integrales para identificar, interpretar, argumentar y resolver problemas del contexto con idoneidad, metacognición y compromiso ético, desarrollando y movilizando el saber ser, el saber hacer y el saber conocer (Tobón, 2010).

Para efectos del presente estudio, fue necesario analizar los tipos de competencias que deben poseer los docentes de secundaria; así, Perrenoud (2008) selecciona un conjunto de competencias, consideradas como el resultado de una construcción teórica conectada a la problemática del cambio que invitan al docente a redescubrirse e inventar, de acuerdo a la realidad de su experiencia y práctica, destacando la competencia de informar e implicar a los padres; manifestada en tareas concretas: favorecer reuniones informativas y de debate, dirigir las reuniones, implicar a los padres en la valorización de la construcción de conocimientos.

Por su parte Marchesi, y Díaz, (2009) explican que en la figura docente se intersectan tres esferas y dentro de ellas, se considera la competencia de cooperar con la familia.

Sin embargo; hasta los actuales momentos, de acuerdo a la literatura consultada, no ha sido lo suficientemente tratada la competencia orientación educativa familiar en los docentes, considerándose ésta como una herramienta de mucha importancia para el logro de los objetivos en la educación, es decir, la formación integral y armónica de los estudiantes para lo que se requiere indiscutiblemente de la participación de la familia, siendo ineludible que el docente cuente con los saberes que le permitan orientar a la misma en su función educativa. 


\section{Condicionantes sociales para la formación y desarrollo de la competencia orientación educativa familiar en los docentes de educación básica superior}

En la mayoría de los países, las reformas educativas por lo general son impuestas desde arriba, entregadas a los maestros y centros educativos para su aplicación; estas reformas tienden a concebirse como una rápida reingeniería, no como un proceso de aprendizaje social que involucra a muchos actores, sin considerar en la mayoría de los casos que el éxito y continuidad de los cambios dependen, en última instancia de los maestros, de su voluntad, de sus conocimientos y de su real aplicación en la cotidianidad de la gestión educativa.

En Ecuador, se ha vivido, en varias ocasiones estas experiencias donde se implantan reformas educativas, casi al mismo tiempo que son reemplazadas por otras, generando en todos los actores de la educación cierta incertidumbre en cuanto a su accionar, ya que éstas, de acuerdo al criterio de la autora, en su mayoría no han respondido a las verdaderas realidades del sistema; donde los padres de familia, estudiantes, docentes y autoridades de las instituciones educativas no solo se muestran confundidos ante cambios repentinos, sino estresados por el cumplimiento de los mismos, ya que de acuerdo al Plan Decenal (2006) en la mayoría de los casos existe la exigencia a la aplicación, pero no existe la formación oportuna para tal efecto, sin considerar incluso que el $17.98 \%$ de los profesores que se encuentran en servicio, no tiene título docente, por lo que no se puede asumir que sabrán cómo aplicar los cambios que se imponen.

La eficacia de la educación se ve reflejada en sus consecuencias y resultados; así, los resultados de medición de la calidad de la educación en Ecuador, según el Banco Mundial, presentado en el Plan Decenal (2006) son los más bajos entre 19 países latinoamericanos; por otra parte, las pruebas APRENDO que se aplicaron en los años 1996, 1998 y 2000, cuyos resultados demostraron que en las habilidades fundamentales como son dominio del lenguaje y de la matemática, los niños ecuatorianos tienen problemas ya que los promedios son muy bajos (ninguno de los promedios superó los doce puntos), lo que denota la mala calidad de la enseñanza en estas materias instrumentales base del desarrollo de la inteligencia (de acuerdo al mismo informe). 
Frente a esta realidad, el Ministerio de Educación ha desarrollado programas de mejoramiento profesional a través de la capacitación docente; en los actuales momentos, la Subsecretaría de Desarrollo Profesional Educativo, con su programa Sistema Integral de Desarrollo Profesional para Educadores -SÍPROFE, despliega cursos que tienen como objetivo el desarrollo de destrezas pedagógicas comunes, con temas como Pedagogía, Didáctica, uso de las TICs, pero se puede denotar que no se ejecutan talleres de capacitación en temas relacionados con el desarrollo de competencias específicas en los docentes.

Para nadie es desconocido que los docentes, especialmente los que laboran con niños y adolescentes, deben mantener comunicación permanente con los padres de familia y/o representantes ya que través de esa comunicación permite fortalecer la relación escuela familia, con el objetivo de obtener el apoyo de los padres en la tarea de educar integralmente a sus hijos y en colaboración mutua superar las debilidades antes mencionadas.

Existe un vínculo entre una buena relación familia- escuela y una enseñanza escolar de calidad, además afirma que existe una conexión entre los fracasos escolares, culturales y sociales al distanciar la escuela con el hogar. El profesorado que se interese más por la vida social y familiar de sus estudiantes aumenta la motivación de éstos, ya que obtiene más información para tomar mejores decisiones a la hora de planificar la educación. El conocimiento de la vida personal y experiencia cultural de los estudiantes es una buena base para un buen y acertado proceso de aprendizaje (Villarroel y Sánchez, 2002)

A pesar de ello, la mayoría de los docentes no poseen las herramientas necesarias para cumplir con esta función, en muchas ocasiones esa relación es distante y muy formal, ya que consiste solo en informar sobre desempeño de los estudiantes, sobre todo de los aspectos negativos, o el envío de esquelas informativas; determinándose como un verdadero problema social que los docentes no están preparados para el desempeño en la competencia orientación educativa familiar, a tal punto, que en ocasiones muestran relaciones de contrarios.

Al profundizar en esta problemática, resulta imperante resaltar el papel fundamental que juegan los padres y maestros en la calidad de la educación, considerando que la relación familia - escuela es en mucho una materia de actitudes. Los docentes juegan un papel muy importante, en muchas ocasiones decisivo en el desarrollo de una buena relación. 
El profesorado tiene el poder institucional y ciertos padres pueden sentirse inferiores a ellos, pensando incluso que su intervención puede llegar a perjudicar a los hijos. Los padres rara vez experimentan apoyo social por parte de la escuela, aunque el profesorado diga que lo hace. Fomentar práctica desde los profesores hacia los padres, para darles autoconfianza es por lo tanto muy importante. La comunicación basada en el apoyo social, el elogio, y el ánimo, es crucial; la retroalimentación hacia los padres debería ser acertada y precisa para evitar malos entendimientos (Hernández, 2006).

Los padres y madres que sienten que juegan un papel importante ayudando a sus hijos e hijas en el rendimiento académico y se involucran a sí mismos en su educación, pueden experimentar cambios en ellos mismos.

Del análisis efectuado se deducen serias necesidades sociales, que demandan la intervención inmediata, que permita subsanar en parte la gran problemática social registrada anteriormente. Esa intervención se deberá sustentar desde la ciencia y la tecnología; puesto que la competencia de orientación educativa a la familia se clasifica como tecnología social con impacto educativo, considerando que la tecnología educativa se trata de un modo sistémico de concebir, aplicar y evaluar la totalidad del proceso educativo en función de unos objetivos precisos, basados en investigaciones referentes a la instrucción y la comunicación humana, que utilizan un conjunto de medios humanos y materiales con el fin de dispensar una educación más eficaz (Núñez y Palacios, s.n)

Con la formación y desarrollo en la competencia orientación educativa familiar propuesta por la autora de esta investigación, se obtienen resultados tales como: la calidad de la educación mejorará considerablemente, la institución educativa fortalecerá su relación escuela - familia, los padres se sentirán seguros y apoyados por la labor orientadora que realizan los maestros, Mejoramiento de los niveles de comunicación, los estudiantes se sentirán seguros y apoyados evidenciados en su comportamiento y desempeño académico, las familias de los mismos docentes serán fortalecidas, ya que deberán actuar coherentemente con sus propias familias, las relaciones interpersonales en la comunidad educativa se desarrollarán en un clima de respeto y consideración mutua, desarrollo del trabajo en equipo de la institución educativa, los docentes tendrán un conocimiento individualizado de sus estudiantes, y se fortalecerá el trabajo coordinado y colaborativo con la intervención de otras áreas y profesionales de la institución educativa. 


\section{Políticas sobre las que se sustenta la competencia}

Los profesores son el motor fundamental de los cambios de largo alcance en la educación y su desarrollo profesional es indispensable para obtener los logros esperados en los sistemas educativos. Las políticas que guiarán la ejecución de la nueva generación de reformas educativas en el presente siglo tendrán que centrarse en el fortalecimiento de la escuela y de la capacidad de aprendizaje de quienes van a enseñar a otros a aprender a través de toda la vida.

La formación de los docentes en los diferentes aspectos que le permitan desarrollar su actuación, se sustenta indiscutiblemente en todas las esferas y en políticas establecidas, de esa forma, en el Plan Decenal de Educación 2006-2015 (2006), como parte de la política 7 se incluye la revalorización de la profesión docente y su desarrollo profesional, indicando que un factor que contribuye significativamente en los procesos de mejoramiento de la calidad de la educación es el docente; de la misma manera en el Plan Nacional para el Buen Vivir, que se propone como objetivo 2, "mejorar las capacidades y potencialidades de la ciudadanía que requieren de acciones armónicas e integrales en cada ámbito".

Por su parte la Ley Orgánica de Educación Intercultural (2011), el Código de la Niñez y la adolescencia (2003), el Ministerio de Educación a través de la Subsecretaría de Desarrollo Profesional Educativo, en relación a la corresponsabilidad, buscan mejorar y potencializar la educación del país, por medio de acciones estratégicas, así como el cumplimiento de objetivos comunes.

Para que las leyes y normativas planteadas en los distintos niveles se hagan vida, es necesario que vayan acompañadas de políticas económicas, que permitan la ejecución de programas y proyectos para el cumplimiento de las metas trazadas; de esa forma, se puede mencionar que a nivel del país, el gobierno nacional propone el cambio definitivo de la matriz productiva, es decir, pasar de un capitalismo industrial a un capitalismo cognitivo, para ello apuntalará el talento humano a través del conocimiento, la investigación y la educación, generando una serie de alternativas como la promoción de becas y facilidades de estudios para estudiantes y docentes. 


\section{Materiales y métodos}

Para el desarrollo de la experiencia, se realizó una investigación de campo. Esta consistió en la aplicación de encuestas a docentes y padres de familia de la Unidad Educativa ITSUP de la ciudad de Portoviejo. También fue de mucha importancia la realización de la observación directa de acciones emprendidas en la institución, donde se evidenció la efectividad de la relación de los profesores con la familia.

\section{Resultados}

En el cuadro 1 se hace referencia a las actividades que realizan los docentes en su gestión relación escuela - familia y que según Berzosa et al. (2000) las complicaciones de una adecuada coordinación padres-profesores son en buena medida las responsables que aún no se haya podido avanzar lo suficiente; es el terreno en el que falta un plan por perfeccionar, lo que precisa la necesidad de la formación de competencias en los docentes dentro de las que se encuentra la orientación educativa familiar.

Cuadro 1. Actividades que realizan los docentes en su gestión relación escuela-familia

\begin{tabular}{|c|c|c|c|c|}
\hline Actividades & Siempre & $\begin{array}{l}\text { Casi } \\
\text { siempre }\end{array}$ & $\begin{array}{l}\text { Algunas } \\
\text { veces }\end{array}$ & Nunca \\
\hline $\begin{array}{l}\text { Planifico, organizo, ejecuto y controlo el proceso de enseñanza- aprendizaje } \\
\text { de la asignatura que imparto en función de la formación integral de mis } \\
\text { estudiantes. }\end{array}$ & 26 & 64 & 10 & - \\
\hline $\begin{array}{l}\text { Realizo una caracterización psicopedagógica de mis alumnos y le doy } \\
\text { seguimiento. }\end{array}$ & - & - & 87 & 13 \\
\hline $\begin{array}{l}\text { Busco las vías de solución a los conflictos que se presentan en mi actividad } \\
\text { como maestro. }\end{array}$ & 8 & 9 & 83 & - \\
\hline Informo a los padres de familia de la situación académica de sus hijos. & 10 & 43 & 47 & - \\
\hline $\begin{array}{l}\text { Me siento preparado para enfrentar los conflictos que se me pueden } \\
\text { presentar con los padres de familia. }\end{array}$ & 5 & 17 & 40 & 38 \\
\hline $\begin{array}{l}\text { Oriento a los padres de familia para la solución de problemas académicos y } \\
\text { conductuales de sus hijos. }\end{array}$ & 6 & 13 & 32 & 49 \\
\hline Oriento a los padres de familia para la educación de sus hijos. & 5 & 5 & 43 & 47 \\
\hline
\end{tabular}

Fuente: Docentes de la Unidad Educativa ITSUP

En el cuadro se evidencia que en la Unidad Educativa ITSUP, el 64\% de docentes planifica, organiza y ejecuta el proceso de enseñanza aprendizaje de sus asignaturas. De la misma manera el $87 \%$ de profesores algunas veces realiza la caracterización psicopedagógica de sus alumnos y les da seguimiento. También se observa que el $83 \%$ busca vías de solución a los conflictos que se les presentan. 
La información sobre las situaciones académicas de los docentes a los padres de familia oscila de la categoría de casi siempre representada por $43 \%$ a la categoría inferior de algunas veces, la cual arrojó un $47 \%$. De la misma forma se ilustra en el cuadro que el $38 \%$ de los docentes no se siente preparado para enfrentar los conflictos que se pueden presentar con los padres de familia. Se visualizó que el $49 \%$ de maestros no orienta a los padres para la solución de problemas académicos y conductuales de sus hijos, probándose además que el $43 \%$ de estos no realiza la orientación educativa a la familia.

En el cuadro 2, sobre las acciones y actitudes de los padres de familia en relación con su rol educativo, Valdés et al. (2009) manifiestan que los estudiantes en todos los niveles hacen el mejor trabajo académico y tienen actitudes escolares más positivas, aspiraciones más altas y otros comportamientos positivos, si tienen padres conscientes del valor de la escuela y de los logros académicos de los hijos.

Cuadro 2. Acciones y actitudes de los padres de familia en relación a su rol educativo

\begin{tabular}{|c|c|c|c|c|}
\hline Actividades & Siempre & $\begin{array}{l}\text { Casi } \\
\text { siempre }\end{array}$ & $\begin{array}{l}\text { Algunas } \\
\text { veces }\end{array}$ & Nunca \\
\hline $\begin{array}{l}\text { Considero que las actividades de orientación educativa } \\
\text { familiar son importantes }\end{array}$ & - & 80 & 20 & - \\
\hline $\begin{array}{l}\text { Dedico tiempo a las actividades de orientación educativa } \\
\text { familiar que desarrolla la institución }\end{array}$ & 13 & 26 & 40 & 21 \\
\hline $\begin{array}{l}\text { Se siente motivado por participar en las actividades de } \\
\text { orientación que se desarrollan en la Unidad Educativa }\end{array}$ & 21 & 47 & 29 & 3 \\
\hline $\begin{array}{l}\text { Considera que todos los profesores de su hijo } \\
\text { contribuyen a su preparación para lograr la correcta } \\
\text { formación de sus hijos }\end{array}$ & - & 27 & 40 & 33 \\
\hline
\end{tabular}

Fuente: Padres de Familia de la Unidad Educativa ITSUP

En el cuadro se evidencia que para los padres de familia de la Unidad Educativa ITSUP en un $80 \%$ las actividades de orientación educativa familiar son importantes; sin embargo el $40 \%$ solo algunas veces dedica tiempo para este tipo de actividades que desarrolla la institución. Adicionalmente el $47 \%$ de padres encuestados manifiesta que casi siempre se siente motivado a participar en las actividades de orientación que se desarrollan en el plantel. También se demuestra que de acuerdo a la opinión de los padres, representado en el $40 \%$ considera que no todos los profesores contribuyen a su preparación para lograr la correcta formación de los hijos. Aspecto de trascendental importancia en el mejoramiento de las relaciones escuelafamilia y por ende en la formación integral de los niños y jóvenes. 


\section{Discusión}

Es necesario señalar, entonces, que la familia y la escuela por separado no podrán jamás cumplir con los altos propósitos de la calidad educativa descritos en los párrafos anteriores. Por lo tanto, es necesario propiciar y promover una alianza entre ellas, ya que ambas se necesitan para poder diseñar y aplicar estrategias a favor del desarrollo integral de los niños y jóvenes, considerando que cuando la familia participa en las actividades escolares y se involucra con la tarea escolar de sus hijos e hijas, éstos tendrán más oportunidades de sobresalir académicamente (Korinfeld, 2000)

De acuerdo a los resultados se constata que ninguna familia se desinteresa totalmente de la escolarización de sus hijos e hijas o es indiferente a la institución; sin embargo, algunos pueden evitar la participación activa y las relaciones entre los actores de ambas instituciones (escuela y familia). Otro aspecto que se desprende de los datos anteriores y que se han tenido poco en cuenta en los planteles educativos, es la sensibilización de las familias y de los profesores sobre la necesidad de participar mutuamente en la formación integral de los estudiantes y la formación de cómo hacerlo (Mérida, 2002; López y Alonso, 2011) .Para Marín, et al. (2013). un requisito general y poco atendido es la formación de todos los participantes. Esta formación debe iniciarse con el cuerpo docente que a menudo tiene una imagen negativa del papel educativo de los padres y que si puede hacerlo, mantiene la distancia con ellos (Katzenbach, 2000).

En el análisis efectuado se determina que sin el apoyo diario de la familia es muy difícil que la escuela pueda formar sujetos capaces de respetarse a sí mismos y a los demás y ser también capaces de aprender hábitos y valores necesarios para lograr una mejor calidad de vida (Guevara 2012,Vol 2006, Gomaris y Martínez 2010), entre otros.

\section{Conclusiones}

Según el análisis efectuado de la necesidad y fundamentos epistemológicos de la formación y desarrollo de la competencia orientación educativa familiar en los docentes, se concluyó que tanto la escuela como la familia no asumen totalmente el rol educativo que a cada uno le corresponde en la formación integral de los estudiantes y que existe una desvinculación en las relaciones de ambos. Es necesario que se fomente la práctica de la competencia en los docentes a través de un proceso formativo permanente para que exista un fortalecimiento entre los componentes de la escuela y la familia, trabajándose en la práctica de esta. 


\section{Bibliografía}

1. Berzosa, MP et al. 2009. El reto de la orientación familiar, una realidad que necesita mejorar. Revista Apuntes Psicológicos, 27, (3-2), 441 - 456.

2. Carmona, M. 2008. Hacia una formación docente reflexiva y crítica: fundamentos filosóficos, Revista de teoría y Didáctica de la Ciencias Sociales. Mérida- Venezuela, ISSN 1316-9505, (13), 125 - 146.

3. Escudero, J. 2009. La formación del profesorado de Educación Secundaria: contenidos y aprendizajes docentes. Revista de Educación. 350, 79-103. Universidad de Murcia. Facultad de Educación. Área de Didáctica y Organización Escolar. Murcia, España.

4. García, M \& Maquilòn, J. 2010.El futuro de la formación del profesorado universitario, REIFOP, 14, (1), 17 - 26.

5. Guevara, G. 2012. La orientación educativa y familiar. Su implicación en la formación del profesional universitario en Cuba. Gaceta Médica Espirituana, Univ. Ciencias Médicas Sancti Spíritus, 14, (2), 150-156.

6. Gomaris, M \& Martínez, M. 2010. Principios fundamentales en la orientación familiar, Bloque III Habilidades Sociales y Orientación Familiar, DIGITUM Biblioteca Universitaria, Universidad de Murcia, España.

7. Hernández, MA \& Lòpez, H. 2006. Análisis del enfoque actual de la cooperación padres y escuela. Aula Abierta. ICE Universidad de Oviedo, (87 ), 3-26.

8. Jaik A, et, al, 2011. Desempeño docente y satisfacción laboral en educación superior, VI Foro de Investigación Educativa

9. Katzenbach, J, 2000. El trabajo en equipo ventajas y dificultades. Barcelona España: Ediciones Granica, S.A.

10. Korinfeld, S, 2000. Las agencias de trabajo temporario como puente de inclusión al mercado laboral, 6| Congreso Nacional de Estudios del Trabajo, Argentina

11. López, MM \& Alonso, Y. 2011. La participación de la familia y la comunidad en el proceso de enseñanza-aprendizaje de la escuela primaria en Cuba: una perspectiva diferente, Revista Odiseo, (17).

12. Marchesi, A \& Díaz, T .2009. Desafíos de las TIC. El cambio educativo en Iberoamérica Te los Cuadernos de Comunicación e Innovación, (78).

13. Marín, R et al. 2013. La evaluación de las competencias docentes en el modelo DECA: anclajes teóricos [Versión electrónica], Formación Universitaria, 6, (6).

14. Medina, E. J y D. Barquero. 2012. Competencias profesionales para la práctica docente. Mèxico: Editorial Trillas,S.A, de C.V. 
15. Ministerio de Educación y Cultura. 2006. Hacia el Plan Decenal de Educación del Ecuador 2006-2015. Primera versión resumida

16. Mc Pherson, 2000. Superación y capacitación del personal docente cubano en la esfera de la educación sexual. Revista Desafío Escolar, 4 (11).

17. Núñez, N \& Palacios, P. (s.n) La superación docente continua: algunos criterios para su perfeccionamiento. Revista Iberoamericana de Educación. 1-9. 